\title{
Leserbriefe
}

\section{Tendenz: Psychiatrisierung und Ökonomisierung}

In seinem Artikel "Psychiatrisierung in der ökonomisierten Gesellschaft" in Der Neurologe \& Psychiater, Ausgabe 10/2015, prangerte Dr. Rainer Köchert, Ludwigshafen, die zunehmende Psychiatrisierung von Befindlichkeitsstörungen und Ökonomisierung der Psychiatrie an. Hierzu erreichten uns mehrere Leserbriefe.

\section{Professionelle Arbeitsteilung und keine Doppelbehandlung}

"Sehr geehrter Herr Köchert, vielen Dank, dass sie das Thema Ökonomisierung und dessen gravierende Auswirkungen im Gesundheitswesen aufgegriffen haben. Auch ich finde eine Diskussion diesbezüglich äußerst wichtig und sehe die Gefahr, dass Ärzte sich zu Handlangern und Ausführungsorganen dieser Politik machen lassen. Hinzugefügt werden könnte noch die Diskussion um Justizialisierung und Bürokratisierung, die zu den Folgen der Ökonomisierung gehören und unsere ärztliche Tätigkeit unterminieren. Ich stimme Ihnen voll zu, dass unsere Gesellschaft durch die Ökonomisierung insbesondere im psychischen Bereich immer kränker wird und dringend nach demokratischen und menschlichen Lösungen gesucht werden sollte.

Bis auf die sehr gute Idee, sozialrechtliche Fragestellungen als Kassenleistung zu unterbinden, finde ich Ihre Lösungen und das Fazit zu kurz gegriffen sowie mit einem Rundumschlag gegenüber ihren psychotherapeutisch ausgebildeten und tätigen Kollegen versehen.

Selbst die schwerst psychiatrisch kranken Patienten, aber auch Befindlichkeitsstörungen können besser mit guter psychotherapeutischer Ausbildung behandelt werden. Die psychotherapeutische Ausbildung kommt meiner Meinung bei dem Facharzt für Psychiatrie und Psychotherapie zu kurz. Der Rückgriff auf die Allgemeinmediziner und hausärztlichen Internisten für die effiziente Versorgung der ,leichten psychiatrisch Erkrankten' ist eine Illusion. Leider müssen auch die psychosomatisch tätigen Hausärzte im 5-Minuten-Takt arbeiten, um überhaupt ihre Kosten zu decken. Die Hausärzte mit Zusatztitel Psychosomatik und die Fachärzte mit psychosomatischer Grund- versorgung, die am ehesten für die Versorgung der ,Befindlichkeitsstörungen und psychosomatischen Grunderkrankungen' und meiner Meinung Weitervermittlung zu psychotherapeutischen Kollegen infrage kommen, bekommen die Gespräche nicht bezahlt und werden dafür mit Regressen überzogen.

Der Großteil meiner ambulanten psychotherapeutischen Kollegen behandelt mit fundierten Ausbildungen Menschen mit ,harten' und auch schwereren psychiatrischen Diagnosen. Dass sie dabei die Medikamente von kompetenten Hausärzten und Psychiatern verschreiben lassen, finde ich professionelle Arbeitsteilung und keine Doppelbehandlung. Über die Frage, ob die Behandlung der seelischen Folgen sozialpolitischer und sozioökonomischer Missstände Aufgabe der Psychiater/Nervenärzte oder der Ärzte allgemein ist, lässt sich meiner Meinung kaum diskutieren. Dass sich insbesondere Psychiater und Psychotherapeuten aber dagegen wehren, sich zu Handlangern der Ökonomisierungspolitik machen zu lassen und sich nicht dafür einsetzen lassen, die Menschen so zu beeinflussen, dass sie in diesem System besser funktionieren, finde ich wie Sie äußerst wichtig."

Dr. med. Alexandra Urbas, Ärztin für Psychiatrie und Psychotherapie, Kassel

\section{Auch wir Psychotherapeuten leisten gute Arbeit}

"(...) als psychologische Psychotherapeutin kann ich Ihre Bedenken nur bedingt nachvollziehen. Sicher gibt es gelegentlich eine Überversorgung von leicht erkrankten Personen, und gelegentlich mag ein Kollege einem leicht erkrankten (und daher auch zuverlässigeren!) Patienten den Vorzug geben. Doch die pauschale Aburteilung unseres Berusstandes finde ich persönlich nicht angemessen. Ich denke da zum Beispiel an einen meiner Patienten mit einer Angststörung, der sieben Jahre (!) das Haus nicht verließ - lediglich zu Besuchen bei seinem Psychiater, so etwa zweimal im Jahr, mit Eskorte der gesamten Familie. Während seiner Behandlung bei mir hat er sich einen netten Freundeskreis aufgebaut, sich für ein Studium entschieden und dieses begonnen (...) und mittlerweile ist er fertig und wird sicher eine Stütze der Gesellschaft sein, und ein guter Steuerzahler. Dies ist nur ein Beispiel von vielen, die zeigen, dass auch wir Psychotherapeuten gute Arbeit leisten."

Sonja Mayer, Diplompsychologin, Psychologische Psychotherapeutin (VT), Taunusstein

\section{Das spricht mir aus der Seele}

"(...) vielen Dank für Ihre klaren Worte zu einer krassen Fehlentwicklung in unserem Fachgebiet. Das spricht mir aus der Seele. Klartext ist auch mein bevorzugter Stil. (...)"

Dr. med. Burkhard Voß, Krefeld

\section{Nicht instrumentalisieren lassen!}

"(...) besser hätte man die Problematik kaum abhandeln können, als Sie es gemacht haben. Ich ,tröste' mich damit, dass ich nur noch wenige Jahre bis zur Aufgabe der Praxistätigkeit habe und schreibe in den Briefen an die Hausärzte einfach ,(...) medizinisch ist das Problem nicht zu lösen $(. . .)^{\prime}$ und verweise zum Beispiel bei betrieblichen Konflikt- oder Mobbing-Situationen auf den Betriebsrat, rate zum Einschalten eines Anwaltes (...). Wir sollten uns nicht dazu instrumentalisieren lassen, monatelange AU-Zeiten, EU-Renten und anderes diagnostisch zu begründen und zu rechtfertigen."

Dr. med. G. Stengele, Facharzt für Neurologie und Psychiatrie - Psychotherapie, Achern 